Article

\title{
Three Essays on Stopping
}

\section{Eberhard Mayerhofer}

Department of Mathematics and Statistics, University of Limerick, Limerick V94TP9X, Ireland; eberhard.mayerhofer@ul.ie

Received: 27 September 2019; Accepted: 16 October 2019; Published: 18 October 2019

check for updates

\begin{abstract}
First, we give a closed-form formula for first passage time of a reflected Brownian motion with drift. This corrects a formula by Perry et al. (2004). Second, we show that the maximum before a fixed drawdown is exponentially distributed for any drawdown, if and only if the diffusion characteristic $\mu / \sigma^{2}$ is constant. This complements the sufficient condition formulated by Lehoczky (1977). Third, we give an alternative proof for the fact that the maximum before a fixed drawdown is exponentially distributed for any spectrally negative Lévy process, a result due to Mijatović and Pistorius (2012). Our proof is similar, but simpler than Lehoczky (1977) or Landriault et al. (2017).
\end{abstract}

Keywords: reflected Brownian motion; linear diffusions; spectrally negative Lévy processes; drawdown

MSC (2010): 60J65; 60J75

\section{Introduction}

This paper comprises three essays on stopping.

In Section 2, we compute the Laplace transform of the first hitting time of a fixed upper barrier for a reflected Brownian motion with drift. This expands on and corrects a result by Perry et al. (2004).

In Section 3, we show, by using an intrinsic delay differential equation, that for a diffusion process, the maximum before a fixed drawdown threshold is generically exponentially distributed, only if the diffusion characteristic $\mu / \sigma^{2}$ is constant. This complements the sufficient condition formulated by Lehoczky (1977). By solving discrete delay differential equations, we further construct diffusions, where the exponential law only holds for specific drawdown sizes.

Section 4 uses Lehoczky (1977)'s argument to show that the maximum before a fixed drawdown threshold is exponentially distributed for any spectrally negative Lévy process, the parameter being the right-sided logarithmic derivative of the scale function. This yields an alternative proof to the original one in Mijatović and Pistorius (2012) and is also similar to the one in Landriault et al. (2017).

\section{The First Hitting Time for a Reflected Brownian Motion With Drift}

Let $X$ be a reflected Brownian motion on $[0, \infty)$, with drift $\mu$ and volatility $\sigma$. Then $X$ can be written as

$$
X_{t}=x+\mu t+\sigma W_{t}+L_{t},
$$

where $W=\left(W_{t}\right)_{t \geq 0}$ is a standard Brownian motion, and $L=\left(L_{t}\right)_{t \geq 0}$ is an inon-decreasing process, such that the induced random measure $d L$ is supported on $\{X=0\}$. Itô's formula implies that for any $f \in C_{b}^{2}([0, \infty))$ satisfying $f^{\prime}(0+)=0$, the process

$$
f\left(X_{t}\right)-f(x)-\int_{0}^{t} \mathcal{A}_{y} f\left(X_{s}\right) d s
$$


is a martingale, where $\mathcal{A}_{y}$ is the differential operator, defined by $\mathcal{A}_{y} f(y)=\frac{\sigma^{2}}{2} f^{\prime \prime}(y)+\mu f^{\prime}(y){ }^{1}$

For $\delta \geq-x$, we define the first hitting time:

$$
\tau_{\delta}:=\inf \left\{t \geq 0 \mid X_{t}=\delta+x\right\} .
$$

Since, before reaching the boundary 0 , the process cannot be distinguished from a Brownian motion with drift, we may confine ourselves to computing $\tau_{\delta}$ for barriers $\delta+x$, where $\delta>0$. Our aim is to compute the Laplace transform:

$$
\Psi(\theta ; \delta, x):=\mathbb{E}\left[e^{-\theta \tau_{\delta}} \mid X_{0}=x\right], \quad \theta \geq 0 .
$$

Theorem 1. For $\delta \geq 0$, the Laplace transform of the first hitting time of a reflected Brownian motion with drift $\mu$ and volatility $\sigma$ is given by

$$
\Psi(\theta ; x, \delta):=e^{\frac{\delta \mu}{\sigma^{2}}} \frac{\sqrt{\mu^{2}+2 \theta \sigma^{2}} \cosh \left(\frac{x \sqrt{\mu^{2}+2 \theta \sigma^{2}}}{\sigma^{2}}\right)+\mu \sinh \left(\frac{x \sqrt{\mu^{2}+2 \theta \sigma^{2}}}{\sigma^{2}}\right)}{\sqrt{\mu^{2}+2 \theta \sigma^{2}} \cosh \left(\frac{(x+\delta) \sqrt{\mu^{2}+2 \theta \sigma^{2}}}{\sigma^{2}}\right)+\mu \sinh \left(\frac{(x+\delta) \sqrt{\mu^{2}+2 \theta \sigma^{2}}}{\sigma^{2}}\right)} .
$$

Proof. Pick $\Phi \in C_{c}^{\infty}(\mathbb{R})$, such that $\Phi(\xi)=1$ for $|\xi| \leq x+\delta$. Furthermore, let $\kappa \in \mathbb{R}$; then for any $\theta \geq 0$ and $t \geq 0$, the function

$$
F(t, x):=e^{-\theta t} \Phi(x)\left(e^{-\kappa x}+\kappa x\right)
$$

satisfies $f:=F(t, \cdot) \in C_{b}^{2}$ and $f^{\prime}(0)=0$. According to the introductory notes of this section, the process $F\left(t, X_{t}\right)-\int_{0}^{t} \partial_{s} F\left(s, X_{s}\right) d s-\int_{0}^{t} \mathcal{A}_{y} F\left(s, X_{s}\right) d s$ is a uniformly bounded martingale; therefore, the stopped process

$$
F\left(t, X_{t \wedge \tau_{\delta}}\right)-\left(e^{-\kappa x}+\kappa x\right)-\int_{0}^{t \wedge \tau_{\delta}} \partial_{t} F\left(s, X_{s}\right) d s-\int_{0}^{t \wedge \tau_{\delta}} \mathcal{A}_{y} F\left(s, X_{s}\right) d s
$$

is also a true martingale, which starts at zero, $\mathbb{P}^{x}$ - almost surely. Using the fact that $\Phi\left(X_{t \wedge \tau_{\delta}}\right)=1$, we find that the stopped process satisfies for any $t \geq 0$,

$$
\begin{aligned}
e^{-\theta\left(t \wedge \tau_{\delta}\right)} & \left(e^{-\kappa X_{t \wedge \tau_{\delta}}}+\kappa X_{t \wedge \tau_{\delta}}\right)-\left(e^{-\kappa x}+\kappa x\right)+\theta \int_{0}^{t \wedge \tau_{\delta}} e^{-\kappa X_{s}-\theta s} d s+\theta \kappa \int_{0}^{t \wedge \tau_{\delta}} e^{-\theta s} X_{s} d s \\
& -\mu \int_{0}^{t \wedge \tau_{\delta}}\left(\kappa e^{-\theta s}-\kappa e^{-\kappa X_{s}-\theta s}\right) d s-\frac{\sigma^{2} \kappa^{2}}{2} \int_{0}^{t \wedge \tau_{\delta}} e^{-\kappa X_{s}-\theta s} d s \\
= & e^{-\theta\left(t \wedge \tau_{\delta}\right)}\left(e^{-\kappa X_{t \wedge \tau_{\delta}}}+\kappa X_{t \wedge \tau_{\delta}}\right)-\left(e^{-\kappa x}+\kappa x\right)+\theta \kappa \int_{0}^{t \wedge \tau_{\delta}} e^{-\theta s} X_{s} d s \\
& -\frac{\mu \kappa}{\theta}\left(1-e^{-\theta\left(t \wedge \tau_{\delta}\right)}\right)-\left(\frac{\sigma^{2} \kappa^{2}}{2}-\kappa \mu-\theta\right) \int_{0}^{t \wedge \tau_{\delta}} e^{-\kappa X_{s}-\theta s} d s .
\end{aligned}
$$

Letting $t \rightarrow \infty$, we thus get by optional sampling,

$$
\begin{aligned}
& \left(e^{-\kappa(x+\delta)}+\kappa(x+\delta)\right) \mathbb{E}\left[e^{-\theta \tau_{\delta}} \mid X_{0}=x\right]-\left(e^{-\kappa x}+\kappa x\right)+\theta \kappa \mathbb{E}\left[\int_{0}^{\tau_{\delta}} e^{-\theta s} X_{s} d s \mid X_{0}=x\right] \\
& -\frac{\mu \kappa}{\theta}\left(1-\mathbb{E}\left[e^{-\theta \tau_{\delta}} \mid X_{0}=x\right]\right)-\left(\frac{\sigma^{2} \kappa^{2}}{2}-\kappa \mu-\theta\right) \mathbb{E}\left[\int_{0}^{\tau_{\delta}} e^{-\kappa X_{s}-\theta s} d s \mid X_{0}=x\right]=0 .
\end{aligned}
$$

1 In the language of linear diffusions Borodin and Salminen (2012), $X$ has infinitesimal generator $\mathcal{A}_{y}$ acting on the domain $\mathcal{D}\left(\mathcal{A}_{y}\right)=\left\{f \in C_{b}^{2}([0, \infty)) \mid f^{\prime}(0+)=0\right\}$. 
For the two choices $\kappa \in\left\{\kappa_{-}, \kappa_{+}\right\}$, where

$$
\kappa_{ \pm}:=\frac{\mu \pm \sqrt{\mu^{2}+2 \theta \sigma^{2}}}{\sigma^{2}}
$$

we thus obtain two equations, for two unknown moments,

$$
\begin{aligned}
& \left(e^{-\kappa_{ \pm}(x+\delta)}+\kappa_{ \pm}(x+\delta)+\frac{\mu \kappa_{ \pm}}{\theta}\right) \mathbb{E}\left[e^{-\theta \tau_{\delta}} \mid X_{0}=x\right]+\theta \kappa_{ \pm} \mathbb{E}\left[\int_{0}^{\tau} e^{-\theta s} X_{s} d s \mid X_{0}=x\right] \\
& =\left(e^{-\kappa_{ \pm} x}+\kappa x\right)+\frac{\mu \kappa_{ \pm}}{\theta}
\end{aligned}
$$

Solving this linear system for the involved moments yields the Laplace transform of $\tau_{\delta}$, Equation (1).

Remark 1. This result can also be obtained from a more general result for spectrally negative Léry processes, reflected at an upper barrier (Avram et al. 2017, Proposition 4.B and Section 10.1). In fact, the distribution of $\tau^{\delta}$ is equal in distribution to the first hitting time 0 of the Brownian motion $X_{t}=\delta+\sigma B_{t}-\mu t$, starting at $\delta \geq 0$, reflected at $x+\delta>0$. Its Laplace transform is therefore given by

$$
\psi_{\theta}^{[x+\delta]}(\delta)=e^{\frac{\mu \delta}{\sigma^{2}}} \frac{H(x)}{H(x+\delta)},
$$

where

$$
H(\xi)=\sqrt{2 \theta \sigma^{2}+\mu^{2}} \cosh \left(\frac{\xi \sqrt{2 \theta \sigma^{2}+\mu^{2}}}{\sigma^{2}}\right)+\mu \sinh \left(\frac{\xi \sqrt{2 \theta \sigma^{2}+\mu^{2}}}{\sigma^{2}}\right),
$$

(see Avram et al. (2017), Section 10.1).

Remarks On Perry et al. (2004)

For another "sanity check" of Theorem 4, we compute the Laplace transform Equation (1) independently when $\mu=0$ and $x=0$. In this case, the reflected Brownian motion is equal to $|\sigma B|$ in law, where $B$ is a standard Brownian motion. But then $\tau_{\delta}$ is equal in distribution to

$$
\widetilde{\tau}_{\delta}:=\inf \left\{s>0 \mid B_{s} \in\left\{ \pm \frac{\delta}{\sigma}\right\}\right\}
$$

Now, it is well known that the Laplace transform of $\widetilde{\tau}_{\delta}$ is given by

$$
\mathbb{E}\left[e^{-\widetilde{\theta} \tau_{\delta}} \mid X_{0}=x\right]=\frac{1}{\cosh \left(\frac{\delta}{\sigma} \sqrt{2 \theta}\right)}
$$

which indeed coincides with Equation (1) for $\mu \rightarrow 0$ and $x=0$.

Perry et al. (2004), Formula (5.2), state a different Laplace transforms than our Theorem 4. Letting $\mu \rightarrow 0$ in Perry et al. (2004), Formula (5.2) indeed yields for $\sigma^{2}=1$ and $x=0$,

$$
\mathbb{E}\left[e^{-\theta \tau_{\delta}} \mid X_{0}=x\right]=\frac{1}{\cosh (\delta \sqrt{\theta})}
$$

which contradicts Equation (2). The proof of Perry et al. (2004), Lemma 5.1, cannot be rectified, however, by merely fixing the (obviously) missing factor of $1 / 2$ for $\alpha^{2}$ in the second line of their proof. Indeed, in the same line, they forget a factor $e^{-\kappa W(s)}$ in the second integrand; thus, by inserting special values of $\kappa$ into the process in line 2, one does not get rid of the local-time term, as claimed. 


\section{Diffusions with Exponentially Distributed Gains Before Fixed Drawdowns}

Let $X$ be a diffusion process on the interval $[-a, \infty)$, satisfying the SDE

$$
d X_{t}=\mu\left(X_{t}\right) d t+\sigma\left(X_{t}\right) d W_{t}, \quad X_{0}=0,
$$

where $\mu(x)$ and $\sigma(x)$ are locally Lipschitz continuous functions of linear growth on $[-a, \infty)$, and $\sigma(x)>0$ thereon.

For a threshold $0<\delta \leq a$, we define $M^{\delta}$ as the maximum of $X$, prior to a drawdown of size $\delta$, that is

$$
M^{\delta}=M\left(\tau^{\delta}\right), \quad \text { where } \quad M(t):=\max _{s \leq t} X_{s}, \quad \text { and } \quad \tau^{\delta}:=\inf \left\{t>0 \mid M_{t}-X_{t}=\delta\right\}
$$

We use the abbreviation $\Phi(x):=e^{-2 \int_{0}^{x} \gamma(u) d u}$, where $\gamma(x)=\mu(x) / \sigma^{2}(x)$. The following is due to Lehoczky (1977):

Proposition 1.

$$
\log \mathbb{P}\left[M^{\delta} \geq \xi\right]=-\int_{0}^{\xi} \frac{\Phi(u)}{\int_{u-\delta}^{u} \Phi(s) d s} d u, \quad \xi \geq 0
$$

Caution is needed when interpreting the original paper Lehoczky (1977): Lehoczky uses the letter " $\mathrm{a}$ " for three different objects: The drift $\mu(x)$ is denoted as $a(x)$, while $-a$ is the left endpoint of the interval of the support of $X$; third, the threshold $\delta$ in his paper is also called $a$. An inspection of Lehozky's proof reveals that our more general version with $\delta \leq a$ holds.

In terms of diffusion characteristics, Lehoczky's result holds in a more general context. First, the assumption of locally Lipschitz coefficients are too strong, and can be relaxed. For example, we can relax to Hölder regularity of $\sigma(x)$ of order no worse than 1/2, due to Yamada et al. (1971). In addition, we can allow reflecting or absorbing boundary conditions, thus include reflected diffusions. For instance, Proposition 1 holds for a Brownian motion with drift, starting at 0 and being reflected at $-a$, because the process $X$ cannot hit $-a$ before it reaches a strictly positive maximum, due to strict positive volatility $\sigma(0)>0$.

From Equation (4), it can be seen that when $\mu / \sigma^{2}$ is constant, $M^{\delta}$ is exponentially distributed (the special case for for a Brownian motion with drift is due to Taylor (1975), and independently discovered by Golub et al. (2016)). Mijatović and Pistorius (2012) extended this result to spectrally negative Lévy processes: For those, $M^{\delta}$ is also exponentially distributed, with the parameter being the right-sided logarithmic derivative of the scale function, evaluated at the drawdown threshold.

This section characterizes the exponential law for diffusions:

Theorem 2. The following are equivalent:

1. $\mu(x) / \sigma^{2}(x)$ is a constant on $[-a, \infty)$.

2. For each $\delta>0, M^{\delta}$ is exponentially distributed.

Proof of the Theorem. Sufficiency of the first condition for the second one follows directly from Proposition 1. Suppose, therefore, that for each $0<\delta \leq a$, there exists $\Lambda(\delta)>0$ such that $M^{\delta}$ is exponentially distributed with parameter $\Lambda(\delta)$. Then, due to Equation (4),

$$
\int_{0}^{\xi} \frac{\Phi(u)}{\int_{u-\delta}^{u} \Phi(s) d s} d u=\Lambda(\delta) \xi, \quad \xi \geq 0, \quad \delta \leq a .
$$


By this particular functional form, and, since $\mu / \sigma^{2}$ is continuous, it follows that the functions $\Lambda(\delta)$ and $\Phi(x)$ are continuously differentiable. By differentiating Equation (5) with respect to $\xi$, we have

$$
\Phi(\xi)=\Lambda(\delta) \int_{\xi-\delta}^{\xi} \Phi(u) d u, \quad \xi \geq 0, \quad \delta \leq a,
$$

and differentiating with respect to $\delta$ yields, in conjunction with the previous identity,

$$
\frac{\Phi(\xi-\delta)}{\Phi(\xi)}=-\frac{\Lambda^{\prime}(\delta)}{\Lambda^{2}(\delta)}, \quad \xi \geq 0, \quad \delta \leq a
$$

Therefore, also

$$
\frac{\Phi(\xi)}{\Phi(\xi+\delta)}=-\frac{\Lambda^{\prime}(\delta)}{\Lambda^{2}(\delta)}, \quad \xi \geq 0, \quad \delta \leq a,
$$

and dividing the last two equations yields Lobacevsky's functional equation ${ }^{2}$

$$
\begin{gathered}
\Phi(\xi-\delta) \Phi(\xi+\delta)=\Phi(\xi)^{2}, \quad \xi \geq 0, \quad \delta \leq a, \\
\Phi(0)=1 .
\end{gathered}
$$

Note, $\Phi$ is continuously differentiable, and strictly positive. Hence, by taking derivatives with respect to $\delta$, we get

$$
\frac{\Phi^{\prime}(\xi-\delta)}{\Phi(\xi-\delta)}=\frac{\Phi^{\prime}(\xi+\delta)}{\Phi(\xi+\delta)}
$$

and by setting $\xi=\delta$, we thus have

$$
\Phi^{\prime}(2 \xi)=\alpha \Phi(2 \xi), \quad \Phi(0)=1, \quad 0<\xi \leq a,
$$

where $\alpha=\Phi^{\prime}(0) / \Phi(0) \in \mathbb{R}$. We conclude that for some $\beta \in \mathbb{R}$,

$$
\Phi(\xi)=e^{\beta \xi}, \quad 0 \leq \xi \leq 2 a .
$$

By Equation (7), we can extend the exponential solution to $-a \leq \xi<0$ : By setting $\xi=0$, we indeed have

$$
\Phi(-\delta)=\frac{\Phi^{2}(0)}{\Phi(\delta)}=\frac{1}{e^{\beta \delta}}=e^{-\beta \delta}, \quad 0<\delta \leq a .
$$

Similarly, we can successively extend the validity of Equation (8) to the right, using the functional Equation (7). Now that $\Phi(\xi)=e^{\beta \xi}$ for all $\xi \in[-a, \infty)$ we have, by taking the logarithmic derivative of $\Phi$, that $\mu(x) / \sigma^{2}(x)$ is indeed a constant on $[-a, \infty)$.

Examples of processes for which the running maximum at drawdown is exponentially distributed are the following:

1. $\quad(a=-\infty)$ : Brownian motion with drift $\sigma B_{t}+\mu t$.

2. $(a<\infty)$ : Reflected Brownian motion with drift, reflected at $-a$.

3. Similar examples as in 1 and 2 can be constructed, where $\mu(x) / \sigma^{2}(x)$ is constant. These include reflected diffusions.

However, there are processes that do not satisfy Theorem 2, even though they may exhibit exponentially distributed gains before $\delta$ drawdowns for specific choices of $\delta$. One can, for instance, let $\mu / \sigma^{2}$ be constant only on $[-1, \infty)$, and modify $\mu, \sigma^{2}$ on $[-2,-1)$ in such a way, that the SDE Equation (3) has unique global strong solution. Then, by Proposition 1, for any $\delta<1$ the maximum at

2 See (Aczél (1966) p. 82, Chapter 2 Equation (16)) and the references therein. 
drawdown of size $\delta$ is exponentially distributed. It goes without saying, that there must exist $\delta>1$, for which this is not the case.

Similar, but more sophisticated, examples can be constructed by solving delay differential equations for $\Phi(\cdot)=e^{-2 \int_{0}^{\cdot} \mu(u) / \sigma^{2}(u) d u}$, such that only for a specific threshold $\delta, M^{\delta}$ is exponentially distributed. Equation (6) reads in differential form:

$$
\Phi^{\prime}(\xi)=\Lambda(\delta)(\Phi(\xi)-\Phi(\xi-\delta)), \quad \xi \geq 0,
$$

which is the simplest non-trivial (discrete) delay differential equation. To construct a diffusion process for which the maximum before a drawdown of size 1 is exponentially distributed with parameter one, we set $\Lambda(\delta)=\delta=1$, and we choose a strictly positive continuous function $g(x)$ on $[-1,0]$ satisfying $g(0)=1$. To obtain $\Phi$ on $[0, \infty)$, we solve

$$
\Phi^{\prime}(\xi)=\Phi(\xi)-\Phi(\xi-1), \quad \xi \geq 0,
$$

subject to $\Phi(\xi)=g(\xi)$ for $\xi \in[-1,0]$. This problem has a unique solution with exponential growth. However, if $g$ is not an exponentially linear function (that is, of the form $e^{\lambda x}$ for some $\lambda>0$ ), then $\Phi$ is not, and therefore $\mu / \sigma^{2}$ is not constant. An underlying diffusion process $X$ with $M^{1}$ being exponentially distributed with parameter one can for instance be constructed, by solving SDE Equation (3), where $\sigma=1$ and $\mu=-\frac{\Phi^{\prime}(x)}{2 \Phi(x)}$ on $[-1, \infty)$. Due to Theorem 2, $M^{\delta}$ is, in general, not exponentially distributed.

\section{Lehoczky's Proof for Spectrally Negative Lévy Martingales}

We study in this section the distribution of maximal gains ${ }^{3}$ of processes, prior to the occurrence of a fixed loss $\delta>0$. Golub et al. $(2016,2018)$ claim that for a Brownian motion (the toy model of a fair game), this gain is exponentially distributed, with parameter $\delta$; thus, on average, one gains $\delta$ before experiencing a loss of size $\delta$. This result is independent of the volatility of the Brownian motion. In private communication, Golub (2014) raised the question of whether similar scaling laws hold for other processes, e.g., other diffusion models, or processes with jumps. Such models are useful as benchmark models in the context of certain event-based high-frequency trading algorithms, where the Brownian motion is used as a proxy for an asset, and the location of the maximum suggests the beginning of a trend reversal. ${ }^{4}$

The conjecture that a fair game on average experiences the exact same gain as is lost later on may appear intuitive. And this is indeed the case for many continuous-time martingales, those who are time-changed Brownian motions, with a quadratic variation tending to infinity, along almost every path (because the timing is not relevant here). But it is not true for Lévy martingales, as can be seen from Theorem 4. Nevertheless, the (exponential) distribution of gains, not its parameter, is universal within the class of spectrally negative Lévy processes. Besides, the martingale property is not needed to arrive at this result.

After Theorem 4 was proved in the summer of 2019, F. Hubalek kindly pointed out that the result is, in identical form, preceded by Mijatović and Pistorius (2012). Our proof is, however, similar to the one of Lehoczky (1977), and is therefore an alternative, and simpler one. Finally, we also found a replication of Lehoczky's proof in Landriault et al. (2017), Lemma 3.1, however, this proof is also more difficult than ours due the more general discretization used therein.

3 This random gain is called "overshoot" in Golub et al. (2016). In this section, we refrain from using this terminology due to its established meaning in the field of Lévy processes-it is the discrepancy between a certain threshold, and a jump processes' value, passing beyond that threshold.

4 It goes without saying that the first time this maximum is attained is not a stopping time; otherwise, one could devise arbitrage strategies that short-sell the asset at the maximum. 
We assume, that a Lévy process $X$ is given with downward jumps only but not equal to the negative of a Lévy subordinator and not being a deterministic drift ${ }^{5}$. Such a process is defined by its Lévy exponent

$$
\Psi(\theta):=\frac{1}{t} \log \mathbb{E}\left[e^{\theta X_{t}}\right], \quad \theta>0,
$$

which is of the form

$$
\Psi(\theta)=\mu \theta+\frac{\sigma^{2} \theta^{2}}{2}+\int_{(-\infty, 0)}\left(e^{\theta \xi}-1-\theta \xi 1_{[-1,0)}(\xi)\right) v(d \xi), \quad \theta>0,
$$

with Lévy-Khintchine triplet $\mu \in \mathbb{R}, \sigma \in \mathbb{R}$ and a measure $v(d \xi)$ supported on $(-\infty, 0)$, integrating $\min \left(\tilde{\xi}^{2}, 1\right)$.

The scale function $W$ is the unique absolutely continuous function $[0, \infty) \rightarrow[0, \infty)$ with Laplace transform

$$
\int_{0}^{\infty} e^{-\theta x} W(x) d x=\frac{1}{\Psi(\theta)}, \quad \theta>0
$$

Since the processes lack positive jumps, they can only creep up. This assumption is essential to obtain exit probabilities from compact intervals and also for the main Theorem 4.

Theorem 3. (Bertoin 1996, Theorem VII.8) Let $x, y>0$, the probability that X makes its first exit from $[-x, y]$ at $y$ is

$$
\mathbb{P}\left[\tau_{y}<\tau_{-x}\right]=\frac{W(x)}{W(x+y)} .
$$

For a threshold $\delta>0$, we define $M^{\delta}$ as the supremum of $X$, prior to a drawdown of size $\delta$, that is

$$
M^{\delta}=M\left(\tau^{\delta}\right), \quad \text { where } \quad M(t):=\sup _{s \leq t} X_{s}, \quad \text { and } \quad \tau^{\delta}:=\inf \left\{t>0 \mid M_{t}-X_{t} \geq \delta\right\} .
$$

We are ready to state and proof the main theorem:

Theorem 4. For a spectrally negative Lévy process, the maximal gain $M^{\delta}$ before a $\delta$-loss is exponentially distributed with parameter equal to the logarithmic derivative of the scale function, that is,

$$
\mathbb{P}\left[M^{\delta} \geq \xi\right]=e^{-\frac{W^{\prime}(\delta+)}{W(\delta)} \xi} .
$$

Proof of Theorem 4. The proof is inspired by Golub et al. (2016), however, the exact same idea can be traced back to Lehoczky (1977) in the general context of univariate diffusions processes. Let $A_{k, n}$ be the event that $X$ reaches $k \xi / 2^{n}$ before $-\delta+(k-1) / 2^{n} \xi\left(k=1, \ldots, 2^{n}\right)$. The set $\left\{M^{\delta} \geq \xi\right\}$ can be approximated by $\bigcap_{k=1}^{n} A_{k, n}$, which are decreasing for increasing $n$. In other words,

$$
\left\{M^{\delta} \geq \xi\right\}=\bigcap_{n=1}^{\infty} \bigcap_{k=1}^{2^{n}} A_{k, n} .
$$

Therefore,

$$
\mathbb{P}\left[M^{\delta} \geq \xi\right]=\lim _{n \rightarrow \infty} \mathbb{P}\left[\bigcap_{k=1}^{2^{n}} A_{k, n}\right]
$$

5 This is the natural non-degeneracy condition of Bertoin (1996), Chapter VII to ensure that the process creeps up to any level. 
Due to state-independence of the process (translation invariance) and the Markov property

$$
\mathbb{P}\left[\bigcap_{k=1}^{2^{n}} A_{k, n}\right]=\mathbb{P}\left[A_{1, n}\right] \times \prod_{k=2}^{2^{n}} \mathbb{P}\left[A_{k, n} \mid A_{k-1, n}\right]=\left(\mathbb{P}\left[A_{1, n}\right]\right)^{2^{n}}=\left(\frac{W(\delta)}{W\left(\delta+\xi / 2^{n}\right)}\right)^{2^{n}},
$$

where the last identity follows from Theorem 3 . Since $W$ is differentiable from the right at $\delta$, applying L'Hospital's rule yields

$$
\log \mathbb{P}\left[M^{\delta} \geq \xi\right]=\lim _{n \rightarrow \infty} \log \left(\mathbb{P}\left[A_{1, n}\right]\right)^{2^{n}}=-\xi \frac{W^{\prime}(\delta+)}{W(\delta)} .
$$

Remark 2. Theorem 4 implicitly requires right-differentiability of the scale functions, which is for free, because it can be rewritten as an integral of the tail of some finite measure, see (Bertoin (1996), Chapter VII). However, in many models, full $C^{1}$-regularity is guaranteed (cf. the characterization given by (Kuznetsov et al. (2012), Lemma 2.4)).

\section{Examples}

The scale functions for the below processes are taken from review article of Hubalek and Kyprianou (2011). Throughout this section, $\mathcal{E}(\lambda)$ denotes the exponential distribution with parameter $\lambda>0$.

Example 1 (Compound Poisson Process). Assume we have a compound Poisson process with negative exponentially distributed jumps,

$$
X_{t}=c t-\sum_{k=0}^{N_{t}^{\lambda}} \xi_{k}, \quad \xi_{k} \quad \text { i.i.d. and } \sim \mathcal{E}(\mu), \quad c-\lambda / \mu>0 .
$$

We get

$$
W(x)=\frac{1}{c}\left(1+\frac{\lambda}{c \mu-\lambda}\left(1-e^{-(\mu-\lambda / c) x}\right)\right) .
$$

Clearly, $W \in C^{1}(0, \infty)$

$$
W^{\prime}(x)=\frac{\lambda}{c^{2}} e^{-(\mu-\lambda / c) x}
$$

Therefore, by Theorem 4

$$
M^{\delta} \sim \mathcal{E}\left(\frac{\lambda / c}{e^{\delta(\mu-\lambda / c)}-\frac{\lambda / c}{\mu-\lambda / c}}\right), \quad \lim _{\delta \downarrow 0} \mathbb{E}\left[M^{\delta}\right]=\lambda / c>0, \quad \lim _{\delta \uparrow \infty} \mathbb{E}\left[M^{\delta}\right]=\mu-\lambda / c<\infty .
$$

Unlike the previous example, the following two examples exhibit the same qualitative dependence on the threshold $\delta$, as the standard Brownian motion, where $M^{\delta} \sim \mathcal{E}(1 / \delta)$ : when $\delta \rightarrow 0$, the average maximum at drawdown of size $\delta$ tends to 0 , and when $\delta \rightarrow \infty$, this average goes to infinity.

Example 2 (Brownian motion with drift). A Brownian motion with drift $\mu>0$ and volatility $\sigma$,

$$
X_{t}=\mu t+\sigma B_{t}
$$

has scale function

$$
W(x) \sim e^{-\mu x / \sigma^{2}} \sinh \left(\sqrt{\mu} x / \sigma^{2}\right) .
$$


Hence,

$$
\frac{W^{\prime}(x)}{W(x)}=\frac{-\mu / \sigma^{2} \sinh \left(\sqrt{\mu} x / \sigma^{2}\right)+\sqrt{\mu} / \sigma^{2} \cosh \left(\sqrt{\mu} x / \sigma^{2}\right)}{\sinh \left(\sqrt{\mu} x / \sigma^{2}\right) .}
$$

Therefore, by Theorem 4 (see, e.g., Golub et al. (2016)),

$$
M^{\delta} \sim \mathcal{E}\left(\mu / \sigma^{2}\left(\operatorname{coth}\left(\sqrt{\mu} \delta / \sigma^{2}\right)-1\right)\right) .
$$

Example 3 (Caballero and Chaumont (2006)). This is a Léry process without diffusion component, defined by its Léry measure

$$
v(d \xi)=\frac{e^{(\beta-1) \xi}}{\left(e^{\xi}-1\right)^{\beta+1}}, \quad \xi<0,
$$

where $\beta \in(1,2)$, and its Laplace exponent,

$$
\Psi(\theta)=\frac{\Gamma(\theta+\beta)}{\Gamma(\theta) \Gamma(\beta)}, \quad \theta>0 .
$$

The process exhibits Infinite variation jumps, and drifts to $-\infty$, because $\Psi^{\prime}(0)<0$. The scale function is

$$
W(x)=\left(1-e^{-x}\right)^{\beta-1} .
$$

Using Theorem 4, we thus get

$$
M^{\delta} \sim \mathcal{E}\left(\frac{\beta-1}{e^{\delta}-1}\right), \quad \mathbb{E}\left[M^{\delta}\right]=\frac{e^{\delta}-1}{\beta-1} .
$$

The asymptotic behaviour of the logarithmic derivative of the scale function of a spectrally negative Lévy process can be characterized using the asymptotic behaviour of $W$ and $W^{\prime}$, cf. (Kuznetsov et al. 2012, Chapter 3). For instance, $W(0)=W(0+)=0$, if and only if the process is of infinite variation. In the case of finite variation, we can write the process as $\delta t-J_{t}$, where $J$ is a subordinator; and then $W(0)=1 / \delta>0$. Furthermore, $W^{\prime}(0+)=\infty$, if a diffusion component is present, or if the Lévy measure is infinite. These general findings are consistent with the three examples.

Funding: This research received no external funding.

Acknowledgments: I thank John Appleby, Florin Avram, Huayuan Dong, Friedrich Hubalek, Andreas Kyprianou and two anonymous referees for useful comments.

Conflicts of Interest: The author declares no conflict of interest.

\section{References}

Aczél, János. 1966. Lectures on Functional Equations and Their Applications. Waltham: Academic Press, vol. 19.

Avram, Florin, Danijel Grahovac, and Ceren Vardar-Acar. 2017. The W, Z scale functions kit for first passage problems of spectrally negative Lévy processes, and applications to the optimization of dividends. arXiv. arXiv:1706.06841.

Bertoin, Jean. 1996. Lévy Processes. Cambridge: Cambridge University Press, vol. 121.

Borodin, Andrei. N., and Paavo Salminen. 2012. Handbook of Brownian Motion-Facts and Formulae. Basel: Birkhäuser.

Caballero, Maria Emilia, and Loïc Chaumont. 2006. Conditioned stable Lévy processes and the Lamperti representation. Journal of Applied Probability 43: 967-83. [CrossRef]

Golub, Anton. 2014. Flov Technologies, Zürich, Switzerland. Private communication.

Golub, Anton, Gregor Chliamovitch, Alexandre Dupuis, and Bastien Chopard. 2016. Multi-scale representation of high frequency market liquidity. Algorithmic Finance 5: 3-19. [CrossRef]

Golub, Anton, James B. Glattfelder, and Richard B. Olsen. 2018. The alpha engine: Designing an automated trading algorithm. In High-Performance Computing in Finance. London: Chapman and Hall/CRC, pp. 49-76. 
Hubalek, Friedrich, and Andreas E. Kyprianou. 2011. Old and new examples of scale functions for spectrally negative Lévy processes. In Seminar on Stochastic Analysis, Random Fields and Applications VI. Basel: Springer, pp. 119-45.

Kuznetsov, Alexey., Andreas E. Kyprianou, and Victor Rivero. 2012. The theory of scale functions for spectrally negative Lévy processes. In Léry Matters II. New York: Springer, pp. 97-186.

Landriault, David, Bin Li, and Hongzhong Zhang. 2017. On magnitude, asymptotics and duration of drawdowns for Lévy models. Bernoulli 23: 432-58. [CrossRef]

Lehoczky, John P. 1977. Formulas for stopped diffusion processes with stopping times based on the maximum. The Annals of Probability 5: 601-7. [CrossRef]

Mijatović, Aleksandar, and Martijn R. Pistorius. 2012. On the drawdown of completely asymmetric Lévy processes. Stochastic Processes and their Applications 122: 3812-36. [CrossRef]

Perry, David, Wolfgang Stadje, and Shelemyahu Zacks. 2004. The first rendezvous time of Brownian motion and compound Poisson-type processes. Journal of Applied Probability 41: 1059-70. [CrossRef]

Taylor, Howard M. 1975. A stopped Brownian motion formula. The Annals of Probability 3: 234-46. [CrossRef]

Yamada, Toshio, and Shinzo Watanabe. 1971. On the uniqueness of solutions of stochastic differential equations. Journal of Mathematics of Kyoto University 11: 155-67. [CrossRef]

(C) 2019 by the author. Licensee MDPI, Basel, Switzerland. This article is an open access article distributed under the terms and conditions of the Creative Commons Attribution (CC BY) license (http:// creativecommons.org/licenses/by/4.0/). 